УДК 544.336; 523.4-36

ISSN 1606-867X

DOI: https://doi.org/10.17308/kcmf.2019.21/2359

Поступила в редакцию 24.09.2019

Подписана в печать 15.11.2019

\title{
О физико-химических превращениях с участием воды вблизи температуры $-45^{\circ} \mathrm{C}$
}

\author{
() 2019 Г. С. Бордонский ${ }^{\bowtie}$, А. А. Гурулев
}

Институт природных ресурсов, экологии и криологии СО РАН

а/я 1032, Недорезова, 16-а, 672002 Чита, Российская Федерация

\begin{abstract}
Аннотация. Представлена гипотеза о новом механизме ускорения химических реакций с участием переохлажденной воды вблизи $-45^{\circ} \mathrm{C}$. Гипотеза основана на свойствах второй критической точки воды. Предполагается, что возрастание флуктуации энергии молекул воды приводит к ускорению протекания химических превращений. В эксперименте с использованием нанопористых увлажненных силикатных сорбентов удалось наблюдать реакцию взаимодействия водорода с поверхностью пор при $-45^{\circ} \mathrm{C}$. Химические реакции с участием воды по предполагаемому механизму могут ускоренно протекать на линии Видома при температурах от $-45^{\circ} \mathrm{C}$ до $-53{ }^{\circ} \mathrm{C}$ и в области давлений от 0 до $100 \mathrm{MPa}$.
\end{abstract}

Ключевые слова: переохлажденная вода, вторая критическая точка, линия Видома, ускорение химических реакций.

\section{ВВЕДЕНИЕ}

Согласно современным исследованиям в атмосфере холодных планет, их спутников, в веществе комет обнаруживаются разнообразные сложные химические соединения, в том числе необходимые для существования жизни. Например, на поверхности Титана, спутника Сатурна, найден винилцианид, который может образовывать мембраны живых клеток [1], а на комете Чурюмова-Герасименко обнаружены десятки молекул органических соединений [2]. Возникает вопрос, каким образом при температурах существенно ниже $0{ }^{\circ} \mathrm{C}$ могут образовываться такие соединения. В ряде работ высказано предположение, что они образуются при фотохимических реакциях, которые приводят к появлению сложных соединений в атмосфере или на поверхности холодных космических объектов. Кроме фотохимических реакций предполагается и другой их источник - некоторые соединения могут быть индикаторами ранее существовавшей жизни. Например, метан в атмосфере Марса $[3,4]$, который пополняется при выделении из глубин планеты.

Вместе с тем, давно известны различные механизмы ускорения низкотемпературных хими-

Бордонский Георгий Степанович, e-mail: lgc255@mail.ru ческих реакций [5]. Один из них связан с концентрированием растворов при вымерзании воды. Скорость большинства химических реакций при термической активации реагентов определяется уравнением Аррениуса, то есть при понижении температуры она экспоненциально падает. При сверхнизких температурах может преобладать квантово-механическое туннелирование частиц через энергетический барьер. Однако были найдены реакции, скорость которых может увеличиваться при понижении температуры, вопреки простым механизмам термической активации и концентрирования. Объяснение эффекта связывается с многостадийностью реакций, когда каждая константа скорости отдельной реакции положительна, а суммарная оказывается отрицательной [5].

На скорость реакций влияют активация частиц электроразрядом, взрывом, взаимодействием молекул со стенками сосудов, при радиационном воздействии и фазовых переходах. В случае воды и ее растворов существенную роль играют моменты структурных превращений среды $[5,6$, $7,8]$. Например, ускорение коррозии металлов во льду может происходить благодаря высвобождению энергии при рекомбинации структурных дефектов льда [7]. Повышенная химическая активность имеет место на свежеобразованных

Контент доступен под лицензией Creative Commons Attribution 4.0 License.

The content is available under Creative Commons Attribution 4.0 License. 
твердых поверхностях при низких температуpax [9]. Кроме активации частиц при внешних энергетических воздействиях обнаружены и исследуются высокоупорядоченные наносистемы, образованные, например, при низкотемпературной конденсации паров металлов [10].

Вполне вероятно, что возможны и другие механизмы активации молекул, приводящие к ускорению химических реакций при низких температурах. В частности, можно предположить особый механизм образования газовых или жидких компонентов при их синтезе с участием воды в области низких температур на поверхности или приповерхностных областях пористых сред, который определяется особыми свойствами глубоко переохлажденной воды.

Цель настоящей работы - рассмотреть гипотезу о возможном ускорении химических превращений с участием жидкой поровой воды, близкой по свойствам объемной воде, в области температур от -45 до $-53{ }^{\circ} \mathrm{C}$ и давлениях от 0 до $100 \mathrm{MPa}$, определяемом влиянием второй ее критической точки.

\section{СВОЙСТВА ПЕРЕОХЛАЖДЕННОЙ ВоДЫ}

При невысоких давлениях жидкая вода превращается в твердое состояние при температуре вблизи $0{ }^{\circ} \mathrm{C}$. На фазовой диаграмме состояния воды наинизшая температура ее замерзания составляет $-92{ }^{\circ} \mathrm{C}$ при давлении $200 \mathrm{MPa}$ [11]. Хорошо известно, что вода может быть переохлаждена, находясь в метастабильном состоянии, при давлении 0.1 MPa до температуры, приблизительно равной $-37^{\circ} \mathrm{C}$ [12]. Область температур от -37 до $-120^{\circ} \mathrm{C}$ называют “no man's land” (недоступная область), так как в настоящее время не существует технологий получения такой воды.

Вместе с тем, экспериментально установлено, что незамерзшая вода существует в поровом пространстве твердых тел. Из-за влияния энергии поверхности раздела сред она находится в равновесном состоянии, и температура фазового перехода в лед смещается в область отрицательных температур. Однако свойства такой воды могут существенно отличаться от объемной воды из-за влияния поверхности раздела, а температура ее замерзания зависит от геометрии пор и химических особенностей поверхности их материала. Особыми свойствами обладает незамерзшая вода в порах нанометровых размеров, где имеет место сильное влияние поверхности пор на структуру жидкости, захваченной в поровое пространство [13]. Например, для силикатного материала SBA-15 с наноразмерными порами цилиндрической формы вода не замерзает в порах диаметром 2 nm до температуры $\sim-100{ }^{\circ} \mathrm{C}$ [14]. В зависимости от расстояния до поверхности раздела свойства слоев воды могут существенно изменяться. В эксперименте, так и при компьютерном моделировании, сходство или отличие поровой воды от объемной определяется по параметрам структуры (среднему числу водородных связей на молекулу, радиальной функции распределения, спектрам комбинационного рассеяния, дифракционной картине рассеяния нейтронов и рентгеновского излучения, ЯМР и другим параметрам).

Таким образом, жидкая вода может существовать как в поровом пространстве различных материалов до $-100{ }^{\circ} \mathrm{C}$ и более низких температур, так и в макробъемах до -92 C при приложении высоких давлений. В настоящей работе в рамках высказанной гипотезы рассматриваются метастабильная переохлажденная объемная вода, так и поровая вода, близкая по своим характеристикам к объемной воде.

\section{ОБОСНОВАНИЕ ГИПОТЕЗЫ}

Весьма интересно, что в последние десятилетия были обнаружены уникальные физико-химические свойства переохлажденной воды. При компьютерном моделировании найдена вторая ее критическая точка $[15,16]$, она соответствует температуре $-53^{\circ} \mathrm{C}$ и давлению около $100 \mathrm{MPa}$ [14]. Из этой точки на фазовой диаграмме температура - давление исходит линия Видома, характеризующая особое состояние воды, при котором резко возрастают флуктуации плотности и энтропии [17]. Этот эффект приводит к существенному возрастанию теплоемкости жидкой воды при постоянном давлении, ее изотермической сжимаемости и других термодинамических и электрофизических параметров. При атмосферном давлении это имеет место в окрестности $-45{ }^{\circ} \mathrm{C}$ [18]. Фазовая диаграмма холодной воды из [18] представлена на рис. 1.

Как известно, скорость допустимых законами термодинамики химических превращений определяется рядом параметров, в том числе энергетическим барьером реакции, и она при тепловой активации падает по экспоненте при понижении температуры (в соответствии с формулой Аррениуса). Вместе с тем, возрастание флуктуаций энтропии и плотности может приводить к локальному усилению флуктуаций энергии молекул и, следовательно, можно предполо- 
жить, - к ускорению химических превращений в реакциях с участием молекул воды. Рост флуктуаций энергии молекул воды приводит к возрастанию доли молекул, преодолевающих активационный барьер реакции. При этом обратная реакция существенно замедлена, так как образующиеся в результате реакции молекулы не подвергаются усилению флуктуаций.

\section{ЭКСПЕРИМЕНТ}

a) Взаимодействие водорода с поверхностью пор силикагеля. В связи с данным предположением был выполнен эксперимент, в котором поры высушенного силикатного сорбента - силикагеля заполняли газообразным водородом при комнатной температуре с последующим медленным охлаждением среды до температуры $-130{ }^{\circ} \mathrm{C}$.

Методика измерений. Исследовали тепловые и микроволновые свойства среды - вариации температуры и электромагнитных потерь при плавном охлаждении и нагревании камеры, в которой находился образец.

Использовали силикагель КСКГ со средним размером пор 6-8 nm, а также силикагель для хроматографии Acros со средним размером пор 4-6 nm. Для получения сухого силикагеля его перед экспериментом выдерживали при температуре $140-160{ }^{\circ} \mathrm{C}$ в течение нескольких часов. Материал помещали в волновод с сечением $3.4 \times 7.2 \mathrm{~mm}$ и длиной $80 \mathrm{~mm}$ с сетчатой верхней стенкой для проникновения в силикагель газообразного водорода, которым заполняли объем специальной камеры, где образец выдерживали в течение суток. Чистота водорода $99.999 \%$.

Микроволновое излучение регистрировали при помощи широкополосного модуляционного детекторного радиометра миллиметрового диапазона на диоде с барьером Шоттки, а излучение осуществляли перестраиваемым в интервале частот 34-37 GHz генератором на диоде Ганна с мощностью $10 \mathrm{~mW}$. В отсутствии силикагеля в волноводе отношение сигнал/шум около $10^{5}$ при постоянной времени синхронного детектора $0.1 \mathrm{~s}$. Частоту генератора контролировали анализатором спектра. Приборы разработаны в лаборатории геофизики криогенеза ИПРЭК СО РАН.

Регистрацию тепловых особенностей осуществляли с использованием метода термометрии и программируемой климатической камеры Espec SU-261, в которой задавали линейное изменение температуры во времени. Температуру образца измеряли термопарным измерителем системы сбора информации фирмы «Agilent»,

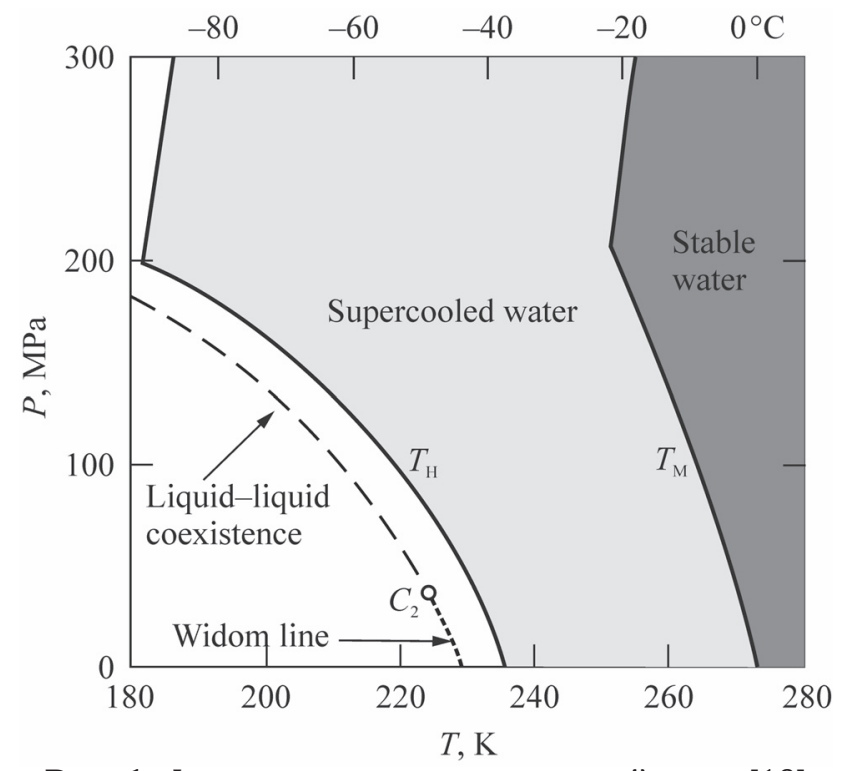

Рис. 1. Фазовая диаграмма холодной воды [18]

[Fig. 1. Phase diagram of cold water [18]]

в которой используется 22 разрядный АЦП. Фазовые переходы первого рода, как и вариации пропускаемой мощности излучения через образец, находили из производных температуры и мощности по времени. Абсолютная точность измерений температуры составляла $1^{\circ} \mathrm{C}$, относительная достигала $0.3{ }^{\circ} \mathrm{C}$ из-за используемого усреднения значений по десяти точкам. Скорость записи данных равнялась трем измерениям в секунду, что составляло массив данных по каждому каналу около 8000 значений за два часа измерений при охлаждении образцов до $100{ }^{\circ} \mathrm{C}$. При усреднении измеряемых величин за три секунды температура образца вдали от фазовых переходов изменялась за это время на значение порядка $0.01{ }^{\circ} \mathrm{C}$.

Методика эксперимента представлена также в $[19,20]$.

Результаты измерений. Особенность силикагеля заключается в том, что поверхность его пор составлена гидроксильными группами, которые активно адсорбируют молекулы воды из газообразной среды. Причем физико-химические свойства воды в таких порах оказываются близки к свойствам объемной воды [21-23]. Например, в [21] методами молекулярной динамики были определены характеристики слоев воды в зависимости от расстояния от поверхности материала (и общего числа слоев молекул воды). Установлено, что первый слой молекул воды имеет отличные от удаленных слоев характеристики и прочно связан с поверхностью. Второй и последующие слои по своим характеристикам соответствуют объемной воде (то есть метаста- 
бильной воде). Приведены плотность жидкости, среднее число водородных связей на молекулу и другие параметры, по которым определено это сходство. В [22] тот же результат (соответствие свойств объемной и жидкой поровой воды) получен термодинамическими расчетами (для размеров пор 3-8 nm). Однако этот результат справедлив только для силикатных сорбентов (например, силикагеля, MCM-41, SBA-15). Для других материалов требуется специальное изучение структуры воды в слоях вблизи поверхности пор.

Было выполнено исследование более двадцати образцов, в том числе в модифицированной установке, в которой силикагель размещали в плоской кювете и использовали другой генератор на частоте $18 \mathrm{GHz}$. В некоторых экспериментах увлажняли поры растворами солей (хлорида натрия и иодида калия). Повторяемые результаты были получены только для высушенного силикагеля.

В экспериментах с сухим силикагелем при $-45^{\circ} \mathrm{C}$ и окрестности этой температуры порядка одного градуса наблюдали выделение тепла, которое может сопутствовать экзотермическими реакциями или фазовым переходам (рис. $2 a$ ). В данном эксперименте, исходя из обычных представлений, какого-либо фазового перехода не должно было наблюдаться, так как при его проведении использовали высушенный при температуре $140{ }^{\circ} \mathrm{C}$ силикагель. Такой материал может содержать незначительное количество прочно адсорбированных молекул воды, которые не подвержены замерзанию. В эксперименте изучали и слабо увлажненный силикагель, для которого наблюдали пропорциональное уменьшение пика на графике производной температуры по времени вблизи $-45^{\circ} \mathrm{C}$ и исчезновение выделения тепла при весовой влажности выше $12 \%$. В случае заполнения пор другими газами, $\mathrm{Ar}$ и $\mathrm{CO}_{2}$, эффект отсутствовал.

В микроволновых экспериментах измерения мощности микроволнового излучения при его распространении через исследуемые среды были выполнены на частоте 34 GHz. Эта частота находится в области наинизшей линии вращательного спектра молекулы водяного пара. В случае протекания химической реакции возможно проявление электромагнитной активности среды. Такая особенность известна для низкотемпературных реакций, как низкотемпературная хемилюминесценция [9]. При температуре $-45^{\circ} \mathrm{C}$ наблюдали кратковременное усиление мощности излучения (рис. $2 b$ ).
Совокупность полученных данных была интерпретирована, как образование слоя молекул воды на поверхности силикатного материала, то есть протекание химической реакции (в данном случае хемосорбции) при взаимодействии водорода и гидроксильных групп в области фазовой диаграммы на линии Видома. Такой вывод был сделан на основании: а) теплового эффекта с выделением теплоты в процессе охлаждения среды вблизи температуры $-45^{\circ} \mathrm{C}$; б) обнаружения усиления мощности микроволнового излучения на частоте $34 \mathrm{GHz}$ при его распространении в волноводе, заполненном силикагелем и водородом в его порах, достигающем в разных экспериментах 1-4 \%; в) возникновением особенностей измеряемых величин в узком температурном интервале вблизи $-45^{\circ} \mathrm{C}$ в хорошем соответствии с имеющимися теоретическими и экспериментальными данными; г) исчезновении выделения теплоты вблизи $-45^{\circ} \mathrm{C}$ при заполнении пор водой (до 12 \% весовой влажности), то есть при уменьшении активной площади сорбента; то же при замене водорода на аргон и углекислый газ.

Детали предполагаемой реакции требуют специального изучения. Возможно, что теплота в области $-45^{\circ} \mathrm{C}$ выделялась при соединении водорода с гидроксильными группами и последующим замерзанием кластеров воды.

б) Замораживание озерных илов. Кроме экспериментов с газообразным веществом были проведены исследования с замораживанием мелкодисперсных природных озерных отложений - илов, состоящих из глинистых материалов и продуктов разложения органического вещества. Частицы этого материала содержат значительный объем полостей нанометровых размеров, что приводит к существованию в них незамерзшей воды до температур $-50{ }^{\circ} \mathrm{C} . . .60^{\circ} \mathrm{C}$, а также разнообразные растворимые вещества.

Цель эксперимента состояла в поиске ускорения протекания химических превращений в разнообразных дисперсных природных средах с участием воды, возможно при смещении линии Видома в фазовом пространстве давлениетемпература для растворов химических соединений. Например, в [18] приведены результаты теоретического рассмотрения сдвига положения второй критической точки и линии Видома для растворов химических соединений в воде.

Илы замораживались в холодильнике и подвергались многочасовой циклической температурной обработке в указанном выше интервале; далее образцы нагревали до комнатной темпе- 


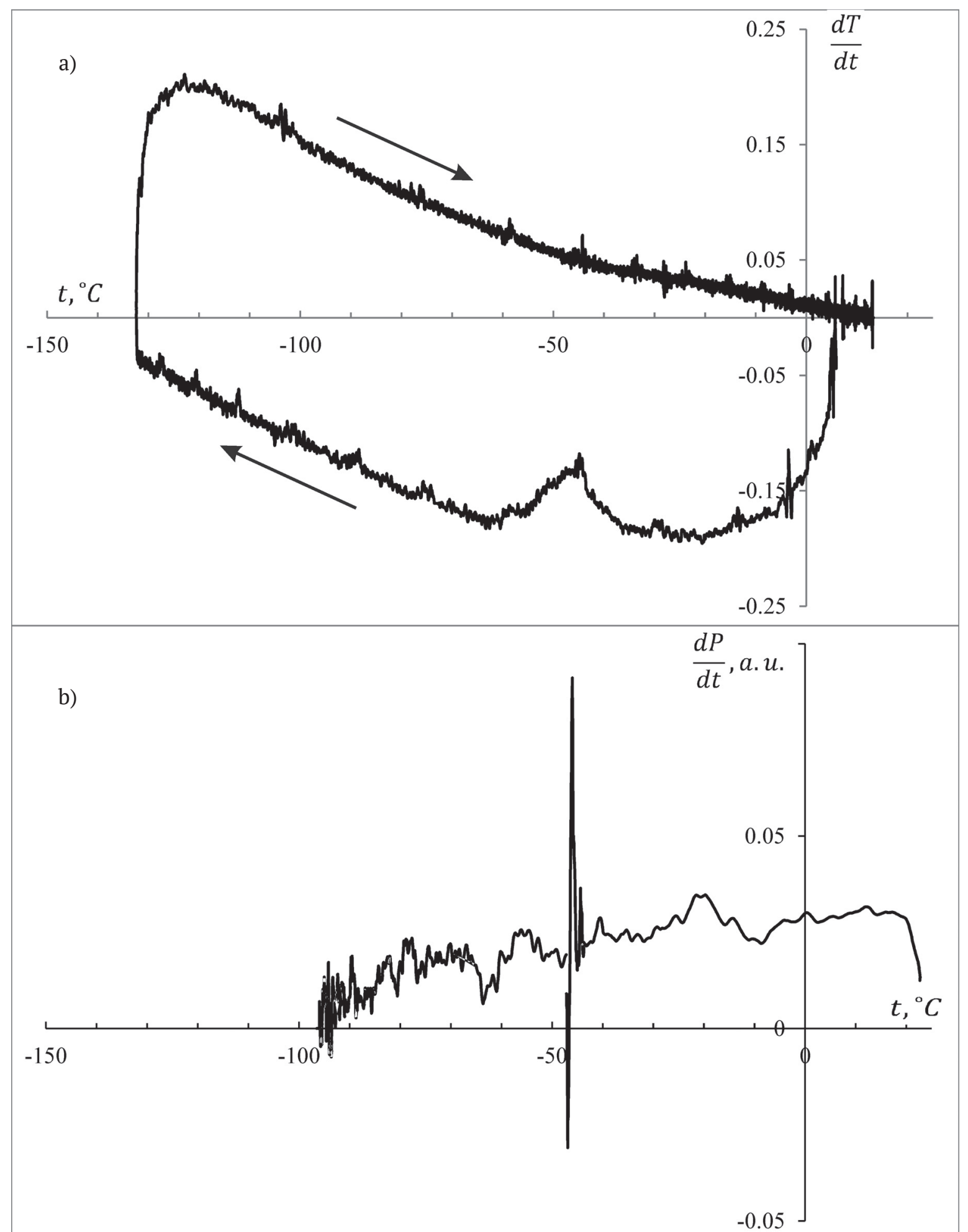

Рис. 2. a) Изменение производной от температуры по времени при термометрии образца высушенного силикагеля КСКГ. $b$ ) График производной проходящей мощности на частоте $34 \mathrm{GHz}$ по времени в зависимости от температуры (образец - сухой силикагель Acros-60 в волноводе со средними размерами пор $6 \mathrm{~nm}$ ). Силикагели насыщены газообразным водородом, скорость охлаждения $-50{ }^{\circ} \mathrm{C} / \mathrm{h}$. Значение производной в относительных единицах. Рис. из работы [19]

[Fig. 2. a) Change in the derivative of temperature over time during thermometry of a sample of dried KSKG silica gel. $b$ ) Time-dependent derivative of transmitted power at a frequency of $34 \mathrm{GHz}$ versus temperature (sample - Acros-60 dry silica gel in a waveguide with an average pore size of $6 \mathrm{~nm}$ ). Silica gels are saturated with gaseous hydrogen, the cooling rate is $-50{ }^{\circ} \mathrm{C} / \mathrm{h}$. The value of the derivative in relative units. Fig. from [19]] 
ратуры и получали водную вытяжку. В ней определяли концентрацию катионов. Точность определения зависела от концентрации катионов и изменялась от десятых долей до десяти процентов. Находили отношение $(N)$ концентрации ионов в вытяжке обработанного вещества и вещества из контрольной группы, не подвергнутой температурной обработке. Полученные результаты для некоторых элементов представлены в табл. 1.

Как следует из табл. 1, наблюдается повышенное значение $N$ ряда элементов в водной вытяжке из илов после их низкотемпературной обработки, так и уменьшение этого отношения ниже единицы.

\section{ОБСУЖДЕНИЕ НИЗКОТЕМПЕРАТУРНОГО МЕХАНИЗМА ХИМИЧЕСКИХ РЕАКЦИЙ}

Полученные предварительные результаты можно интерпретировать, как возрастание скорости химических превращений вблизи температуры $-45^{\circ} \mathrm{C}$ при атмосферном давлении, отличающейся от закона Аррениуса.

Механизм ускорения химических реакций вблизи $-45^{\circ} \mathrm{C}$, как представляется, связан с существенным ростом флуктуаций энтропии и плотности переохлажденной воды на линии Видома. Как известно, при росте флуктуаций в критическом состоянии резко возрастает реакционная способность вещества [24]. Примером являются разработанные технологии уничтожения химических отравляющих веществ. Флуктуации влияют и на фазовые превращения [25]. Следует отметить, что переохлажденная вода на линии Видома является нелинейным объектом, для которого неприменимы законы равновесной термодинамики.

Чисто качественно оценить величину возрастания скорости реакции с участием воды можно следующим образом. Если для данного случая определить значение «эквивалентной» температуры воды $T_{e}[26]$, при которой флуктуации энергии молекул $\Delta E$ равны флуктуациям при $T_{1}=-45^{\circ} \mathrm{C}$, и оно окажется достаточно высо- ким, то это будет являться аргументом в пользу гипотезы.

Оценим значение «эквивалентной» температуры. Это можно сделать, исходя из данных по зависимости теплоемкости при постоянном давлении $\left(C_{p}\right)$ от абсолютной температуры $(T)$. Сначала найдем температуру, при которой флуктуации энтропии равны этой величине при $-45^{\circ} \mathrm{C}$. Среднее квадратичное значение флуктуаций энтропии $\left\langle(\Delta S)^{2}\right\rangle$ связано с теплоемкостью, согласно [27], соотношением: $\left\langle(\Delta S)^{2}\right\rangle=k C_{p}$, где $k$ - постоянная Больцмана. Зависимость удельной $C_{p}$ от $T$ представлена в [18], рис. 3. Как следует из графика рис. 3, теплоемкость жидкой воды по измерениям при стандартном атмосферном давлении имеет экстремум при $-45^{\circ} \mathrm{C}$. Ее значение, согласно расчетам, равно той же величине для насыщенной воды при температуре около $320{ }^{\circ} \mathrm{C}\left(T_{2}\right)$, откуда следует равенство средних квадратов флуктуаций энтропии: $\left\langle(\Delta S)^{2}\left(-45^{\circ} \mathrm{C}\right)\right\rangle=\left\langle(\Delta S)^{2}\left(320^{\circ} \mathrm{C}\right)\right\rangle$. Данный результат указывает на сильное изменение свойств холодной воды при $-45^{\circ} \mathrm{C}$. Однако для оценки ускорения скорости реакции необходимо определить «эквивалентную» температуру, для которой флуктуации не энтропии, а внутренней энергии будут равны ее флуктуациям при $-45^{\circ} \mathrm{C}$.

В качестве искомой величины, характеризующей флуктуации, следует взять стандартное отклонения. Для нахождения стандартного отклонения флуктуаций внутренней энергии $(\Delta E)^{\prime}=\sqrt{\left\langle(\Delta E)^{2}\right\rangle}$ учтем, что $\Delta S=\frac{\Delta E}{T}$ (здесь $T$ берется по шкале абсолютных температур). Обозначим стандартное отклонение флуктуаций энергии при $-45^{\circ} \mathrm{C}$ и искомой $T_{e}$, как $\left(\Delta E_{1}\right)^{\prime}$ и $\left(\Delta E_{e}\right)^{\prime}$.

Значение «эквивалентной температуры» определяется из равенства $\left(\Delta E_{1}\right)^{\prime}=\left(\Delta E_{e}\right)^{\prime}$. Для этого также используем значения удельной теплоемкости из графика рис. 3, для которых найдем отношение величин из формулы Ландау-Лифшица [27] $\sqrt{\Delta S^{2}}=\sqrt{k C_{p}}=\frac{(\Delta E)^{\prime}}{T} ; \sqrt{\frac{C_{1}}{C_{e}}}=\frac{\left(\Delta E_{1}\right)^{\prime} T_{e}}{\left(\Delta E_{e}\right)^{\prime} T_{1}}$

Таблица 1. Отношение концентрации ионов в вытяжке обработанного вещества и вещества из контрольной группы

[Table 1. The ratio of the concentration of ions in the extract of the treated substance and substances from the control group]

\begin{tabular}{|c|c|c|c|c|c|c|c|c|c|c|c|c|c|}
\hline Element & $\mathrm{Sr}$ & $\mathrm{Mg}$ & $\mathrm{S}$ & $\mathrm{Ca}$ & $\mathrm{Na}$ & $\mathrm{Br}$ & $\mathrm{Si}$ & $\mathrm{Zn}$ & $\mathrm{Mn}$ & $\mathrm{B}$ & $\mathrm{Fe}$ & $\mathrm{K}$ & $\mathrm{U}$ \\
\hline$N$ & 3.8 & 3.7 & 3.5 & 3.4 & 3.1 & 2.4 & 1.6 & 1.2 & 1.2 & 1.2 & 0.9 & 0.8 & 0.2 \\
\hline
\end{tabular}

Примечание. Химический анализ проб осуществлялся аналитической лабораторией АО «СЖС Восток Лимитед» (Группа SGS). 
(здесь $C_{1}$ - теплоемкость при постоянном давлении при $-45^{\circ} \mathrm{C}, C_{e}$ - теплоемкость при температуре $\left.T_{e}\right)$. Отсюда при $\left(\Delta E_{1}\right)^{\prime}=\left(\Delta E_{e}\right)^{\prime}$ получаем $T_{e}=T_{1} \sqrt{\frac{C_{1}}{C_{e}}}$. Эта формула справедлива для любой массы объемной воды, так как в ней имеем отношение теплоемкостей.

В области температур $-20^{\circ} \mathrm{C} . . .150{ }^{\circ} \mathrm{C}$ удельная $C_{p}$ изменяется слабо и она приблизительно равна $4.2 \mathrm{~kJ} /(\mathrm{kg} \cdot \mathrm{K})$, а при $-45^{\circ} \mathrm{C}$ эта величина составляет $6.5 \mathrm{~kJ} /(\mathrm{kg} \cdot \mathrm{K})$ (рис. 3). Тогда после вычисления $T_{e}=283 \mathrm{~K}$ (или $10^{\circ} \mathrm{C}$ ).

Таким образом, эквивалентная температура, найденная из равенства значений стандартного отклонения флуктуаций энергии, выше $T_{1}$ на $55^{\circ} \mathrm{C}$. Если использовать найденное приращение при подстановке в формулу закона Аррениуса (для которого скорость реакции возрастает в 2-4 раза при росте температуре на $10^{\circ} \mathrm{C}$ ), то получаем возрастание скорости химических реакций приблизительно в 1000 раз при температуре $-45^{\circ} \mathrm{C}$.

В общем случае при решении задачи об ускорении химических реакций необходимо рассматривать флуктуации свободной энергии Гиббса, а не только внутренней энергии. Так как свободная энергия Гиббса определяется и значениями энтропии, то следует учесть и влияние флуктуаций энтропии. Поскольку температура, при которой флуктуации энтропии равны, достигает $320^{\circ} \mathrm{C}$, можно ожидать и большего возрастания скорости химических реакций, чем это было оценено выше.

Следует отметить, что переохладить объемную воду до $-45^{\circ} \mathrm{C}$ чрезвычайно сложно. Например, экспериментально найденная наинизшая температура переохлаждения жидкокапельных облаков в атмосфере Земли (воды в метастабильном состоянии) составляет значение $-37.5^{\circ} \mathrm{C}$ [12]. Кратковременно, в течение миллисекунд для капель воды микронных размеров при их испарении в вакууме была достигнута температура $-46{ }^{\circ} \mathrm{C}$ [28]. При этом для определения структуры воды (по фактору структурного рассеянии и дифракционной картине) использовали импульсный рентгеновский лазер. В работе [29] при применении метода комбинационного рассеяния света достигнута температура охлажденной жидкой воды, равная $-42.4^{\circ} \mathrm{C}$, для того же метода испарения капель воды в вакууме. В этих исследованиях впервые получена метастабильная объемная вода в области «no man's land» и показана возможность ее су-

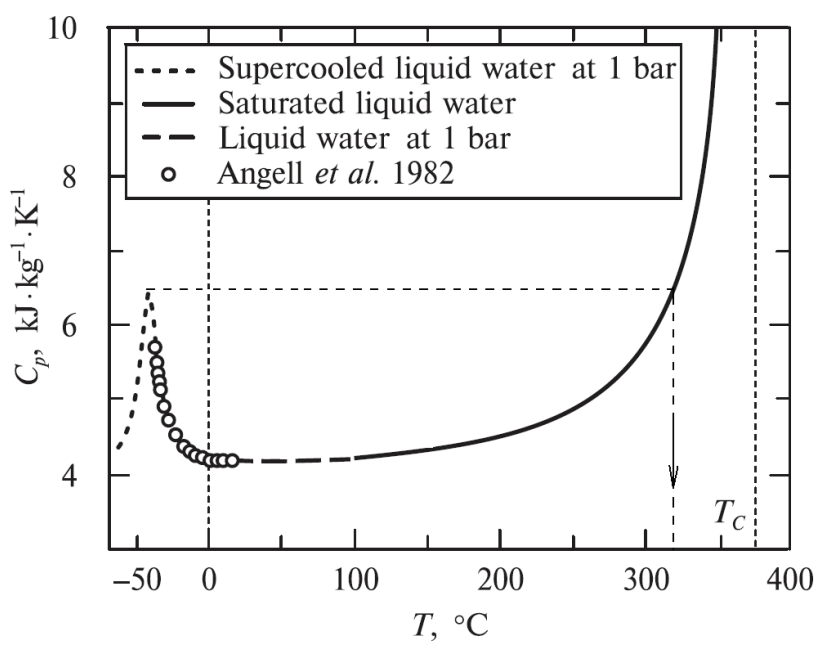

Рис. 3. Теплоемкость жидкой воды при постоянном давлении (из работы [18]). Стрелкой отмечено значение эквивалентной температуры, при которой в переохлажденной воде при $-45^{\circ} \mathrm{C}$ имеют место такие же значения средних квадратичных флуктуаций энтропии и, следовательно, энергии молекул. Экспериментальные точки на графике приведены из статьи [Angell C. A., et al. J. Phys. Chem., 1982, v. 86(6), p. 998].

[Fig. 3. The heat capacity of liquid water at constant pressure (from [18]). The arrow marks the value of the equivalent temperature at which in supercooled water at $-45{ }^{\circ} \mathrm{C}$ the same values of the mean quadratic fluctuations of entropy and, consequently, the energy of molecules take place. The experimental points on the graph are given from the article [Angell C. A., et al. J. Phys. Chem., 1982, v. 86(6), p. 998]].

ществования при $-42{ }^{\circ} \mathrm{C} . .-46^{\circ} \mathrm{C}$. Однако достижение водой, близкой по свойствам объемной воде, температуры $-45^{\circ} \mathrm{C}$ на длительное время возможно в настоящее время только в нанопористых материалах особого химического состава. Но в них при малых размерах пор возникает эффект их закупорки, затрудняющий транспорт жидкостей и газов. Поэтому выход реакций будет уменьшаться и определяться конкретными геометрическими и физико-химическими параметрами увлажненной среды. Кроме того, при рассмотрении данной задачи возникает ряд сложных вопросов о химических процессах для нанометровых объектов, изучение которых недавно начато [30].

\section{ВЫВОдЫ}

Таким образом, есть основания полагать, что в области температур $-45^{\circ} \mathrm{C} . . .-53{ }^{\circ} \mathrm{C}$, для которой наблюдается возрастание теплоемкости при постоянном давлении, имеет место ускорение синтеза химических соединений с участием пе- 
реохлажденной воды. Этот эффект определяется активацией молекул воды на линии Видома из-за возрастания на ней флуктуаций энтропии и энергии.

Кажущаяся виртуальность второй критической точки из-за недоступности температур переохлаждения объемной воды в области «по man's land» оказывается преодолимой как в новых методиках ее получения в метастабильном состоянии, так и для воды в поровом пространстве некоторых материалов (например, силикатных материалов) с размерами пор порядка единиц нанометров. В них возможно охлаждение жидкой воды до $-45^{\circ} \mathrm{C}$ и ниже с сохранением структуры, соответствующей объемной (метастабильной) воде.

Лабораторные эксперименты подтвердили данное предположение на примере образования поверхностного слоя воды из гидроксильных групп поверхности пор и водорода, а также при замораживании увлажненных мелкодисперсных сред - озерных илов.

Грубая теоретическая оценка возрастания скорости протекания химических реакций с участием метастабильной воды при $-45^{\circ} \mathrm{C}$ дает значение ее увеличения приблизительно на три порядка величины. Очевидна необходимость проведения многоплановых экспериментов, так и теоретических исследований. Например, интересен вопрос о смещении положения линии Видома в фазовом пространстве давление-температура для водных растворов тех или иных соединений. Обнаружение такого эффекта, о возможности которого сообщалось в [18], расширит значения критических температур, при которых эффективно протекают криохимические реакции, как в сторону ее понижения, так и повышения.

Возрастание скорости криохимических реакции может осуществляться в атмосфере Земли, ее полярных и субполярных областях, а также на космических объектах, где температура находится в интервале $-45^{\circ} \mathrm{C} . . .-53^{\circ} \mathrm{C}$, а давление составляет $0 \mathrm{MPa} . .100 \mathrm{MPa}$. Например, отмеченные температуры и давления существуют на поверхности Марса, в глубине покровов спутников Сатурна Энцеладе и Титане, а также на других объектах Солнечной системы.

\section{ИСТОЧНИК ФИНАНСИРОВАНИЯ}

Работа выполнена при финансовой поддержке РФФИ (проект №18-05-00085).

\section{КОНФЛИКТ ИНТЕРЕСОВ}

Авторы декларируют отсутствие явных и потенциальных конфликтов интересов, связанных с публикацией настоящей статьи.

\section{СПИСОК ЛИТЕРАТУРЫ}

1. Palmer M. Y., Cordiner M. A., Nixon C. A., Charnley S. B., Teanby N. A., Kisiel, Z., Irwin P. G. J., Mumma M. J. ALMA detection and astrobiological potential of vinyl cyanide on Titan // Science Advances, 2017, v. 3(7), p. e1700022/6. DOI: https://doi.org/10.1126/ sciadv. 1700022

2. Goesmann F., Rosenbauer H., Bredehöft J. H., Cabane M., Ehrenfreund P., Gautier T., Giri C., Kröger H., Le Roy L., MacDermott A.J., McKennaLawlor S., Meierhenrich U.J., Caro G.M.M., Raulin F., Roll R., Steele A., Steininger H., Sternberg R., Szopa C., Thiemann W., Ulamec S. Organic compounds on comet 67P/Churyumov-Gerasimenko revealed by COSAC mass spectrometry // Science, 2015, v. 349(6247), p. aab0689-1/3. DOI: https://doi.org/10.1126/science. aab0689

3. Mumma M. J., Villanueva G. L., Novak R. E., Hewagama T., Bonev B.P., DiSanti M. A., Mandell A. M., Smith M. D. Strong release of methane on Mars in northern summer 2003 // Science, 2009, v. 323(5917), pp. 1041-1045. DOI: https://doi.org/10.1126/ science. 1165243.

4. Кораблев О. И. Исследования атмосфер планет земной группы // Успехи физических наук, 2005, т. 175(6), с. 655-664.

5. Сергеев Г. Б., Батюк В. А. Криохимия. М.: Химия, 1978, 296 с.

6. Шавлов А. В., Писарев А. Д., Рябцева А. А. Динамика электропроводности пленок металлов во льду при его структурном превращении. Рекомбинационно-фононный механизм ускорения коррозии // Криосфера Земли, 2006, т. 10(3), с. 42-48.

7. Шавлов А. В., Писарев А. Д., Рябцева А. А. Коррозия пленок металлов во льду. Динамика электропроводности пленок // Журнал физической химии, 2007, т. 81(7), с. 1180-1185.

8. Великоцкий М. А. Коррозионная активность грунтов в различных природных зонах // Вестник Московского университета. Серия 5: География, 2010(1), c. 21-27.

9. Лотник С. В., Казаков В. П. Низкотемпературная хемилюминесценция. М.: Наука, 1987, 176 с.

10. Шабатина Т. И., Сергеев Г. Б. Реакции при низких температурах в химии наносистем // Усnехи химии, 2003, т. 72(7), с. 643-663.

11. Chaplin M. Water Structure and Science. Режим доступа: http://www.lsbu.ac.uk/water/chaplin.html (дата обращения: 23.09.2019)

12. Rosenfeld D., Woodley W. L. Deep convective clouds with sustained supercooled liquid water down 
to $-37.5^{\circ} \mathrm{C} / /$ Nature, 2000 , v. $405(6785)$, pp. $440-442$. DOI: https://doi.org/10.1038/35013030

13. Бордонский Г. С., Орлов А. О. Признаки возникновения льда “0” в увлажненных нанопористых средах при электромагнитных измерениях // Письма в Журнал экспериментальной и теоретической физики, 2017, т. 105(7-8), с. 483-488. DOI: https://doi. org/10.7868/S0370274X17080045

14. Limmer D. T., Chandler D. The putative liquidliquid transition is a liquid-solid transition in atomistic models of water // Journal of Chemical Physics, 2011, v. 135, pp. 134503/10. DOI: https://doi.org/10.1063/ 1.3643333

15. Mishima O. Volume of supercooled water under pressure and the liquid-liquid critical point // Journal of Chemical Physics, 2010, v. 133(14), p. 144503/6. DOI: https://doi.org/10.1063/1.3487999

16. Handle P. H., Loerting T., Sciortino F. Supercooled and glassywater:Metastable liquid(s), amorphous solid(s), and a no-man's land // Proceedings of the National Academy of Sciences USA, 2017, v. 114(51), pp. 13336-13344. DOI: https://doi.org/10.1073/ pnas. 1700103114

17. Speedy R. J., Angell C. A. Isothermal compressibility of supercooled water and evidence for a thermodynamic singularity at $-45^{\circ} \mathrm{C} / /$ The Journal of Chemical Physics, 1976, v. 65(3), pp. 851-858. DOI: https://doi.org/10.1063/1.433153

18. Анисимов М. А. Холодная и переохлажденная вода как необычный сверхкритический флюид // Сверхкритические флюиды: Теория и практика, 2012, т. 7(2), с. 19-37.

19. Бордонский Г. С., Гурулев А. А. Экспериментальное доказательство существования линии Видома по особенностям поведения водорода в нанопористом силикате при $-45^{\circ} \mathrm{C}$ и атмосферном давлении // Письма в Журнал технической физики, 2017, т. 43(8), c. 34-40.DOI: https://doi.org/10.21883/ PJTF.2017.08.44541.16581

20. Бордонский Г. С., Гурулев А. А., Крылов С. Д., Цыренжапов С.В. Использование микроволновой спектроскопии для изучения состояния переохлажденной воды // Конденсированные среды и межфазные гранищы, 2019, т. 21(1), с. 16-23. DOI: https:// doi.org/10.17308/kcmf.2019.21/712

21. Castrillyn R.V.S., Giovambattista N., Aksay I. A., Debenedetti P. G. Structure and energetics of thin film water // Journal of Physical Chemistry C, 2011,v. 115(11), pp. 4624-4635. DOI: https://doi.org/10.1021/ jp1083967

22. Меньшиков Л. И., Меньшиков П. Л., Федичев П. О. Феноменологическая модель гидрофобных и гидрофильных взаимодействий // Журнал экспериментальной и теоретической физики, 2017, т. 152(6), c. 1374-1392. DOI: https://doi.org/10.7868/ S0044451017120215

23. Cerveny S., Mallamace F., Swenson J., Vogel M., $\mathrm{Xu}$ L. Confined Water as Model of Supercooled Water // Chemical Reviews, 2016, v. 116(13), pp. 7608-7625. DOI: https://doi.org/10.1021/acs.chemrev.5b00609

24. Горбатый Ю. Е., Бондаренко Г. В. Сверхкритическое состояние воды // Сверхкритические флюиды: Теория и практика, 2007, т. 2(2), с. 5-19.

25. Паташинский А. 3., Покровский В. Л. Флуктуационная теория фазовых переходов. М.: Наука, $1982,381 \mathrm{c}$.

26. Пригожин И., Кондепуди Д. Современная термодинамика. От тепловых двигателей до диссипативных структур. М.: Мир, 2002, 461 с.

27. Ландау Л. Д., Лифшиц Е. М. Теоретическая физика. Том. 5. Статистическая физика. Часть 1. М.: Физматлит, 2002, 616 с.

28. Sellberg J. A., Huang C., McQueen T. A., Loh N. D., Laksmono H., Schlesinger D., Sierra R. G., Nordlund D., Hampton C. Y., Starodub D., Deponte D. P., Beye M., Chen C., Martin A. V., Barty A., Wikfeldt K. T., Weiss T. M., Caronna C., Feldkamp J., Skinner L. B., Seibert M. M., Messerschmidt M., Williams G. J., Boutet S., Pettersson L. G. M., Bogan M. J., Nilsson A. Ultrafast X-ray probing of water structure below the homogeneous ice nucleation temperature // Nature, 2014,v. 510(7505), pp. 381-384. DOI: https:// doi.org/10.1038/nature13266

29. Goy C., Potenza M. A. C., Dedera S., Tomut M., Guillerm E., Kalinin A., Voss K.-O., Schottelius A., Petridis N., Prosvetov A., Tejeda G., Fernández J. M., Trautmann C., Caupin F., Glasmacher U., Grisenti R. E. Shrinking of Rapidly Evaporating Water Microdroplets Reveals their Extreme Supercooling // Physical Review Letters, 2018, v. 120(1), p. 015501/6. DOI: https://doi. org/10.1103/PhysRevLett.120.015501

30. Сергеев Г.Б. Нанохимия. М.: Книжный дом «Университет», 2015, 384 с. 


\title{
Regarding Physical and Chemical Transformations with the Involvement of Water Near $-45^{\circ} \mathrm{C}$
}

\author{
@2019 G. S. Bordonskiy ${ }^{\circledR}$, A. A. Gurulev \\ Institute of Natural Resources, Ecology and Cryology, Siberian Branch of the Russian Academy of Sciences \\ POB 1032, 16-a Nedorezova str., 672002 Chita, Russian Federation
}

\begin{abstract}
Objective. A hypothesis about a new mechanism for accelerating chemical reactions involving supercooled water near $-45^{\circ} \mathrm{C}$ is presented. The hypothesis is based on the properties of the second critical point of water at a temperature of $-53^{\circ} \mathrm{C}$ and a pressure of $100 \mathrm{Mpa}$ determined by computer modelling. Its influence extends to a special region of the phase space of cold water - the Widom line, which corresponds to $-45^{\circ} \mathrm{C}$ at atmospheric pressure. On the Widom line, an increase in entropy fluctuations and water density are predicted. It is assumed that an increase in the fluctuation of entropy leads to an increase in the fluctuation of the energy of water molecules and an acceleration of the chemical transformations. However, deep overcooling of bulk water is impossible due to its rapid crystallization below $-37^{\circ} \mathrm{C}$.

Methods. Deep cooling is possible if water is in nanoscale pores. The most convenient medium for this purpose is nanoporous silicate sorbents, in which a significant volume of pore water has parameters close to bulk metastable water. In an experiment using nanoporous moistened silicate sorbents, it is possible to achieve supercooling of water to $-60^{\circ} \mathrm{C}$.

In the performed experiment, thermometry of samples of silicate sorbents filled with hydrogen gas was performed. A chemical reaction was checked for near the Widom line. We also investigated the change in the physical properties of the medium using microwave spectroscopy.

Results. In the experiment, it was possible to observe reactions of the interaction of hydrogen with the pore surface by the release of heat, as well as amplification of microwave radiation at a frequency of $34 \mathrm{GHz}$.

Conclusion. Chemical reactions involving water, according to the proposed mechanism, can accelerate on the Widom line at temperatures from $-45^{\circ} \mathrm{C}$ to $-53^{\circ} \mathrm{C}$ and in the pressure range from 0 to $100 \mathrm{MPa}$.
\end{abstract}

Keywords: supercooled water, second critical point, Widom line, acceleration of chemical reactions.

\section{SOURCE OF FINANCING}

The study was supported by the Russian Foundation for Basic Research (grant No. 18-05-0085).

\section{CONFLICT OF INTEREST}

The authors declare the absence of obvious and potential conflicts of interest related to the publication of this article.

\section{REFERENCES}

1. Palmer M. Y., Cordiner M. A., Nixon C. A., Charnley S. B., Teanby N. A., Kisiel, Z., Irwin P. G. J., Mumma M. J. ALMA detection and astrobiological potential of vinyl cyanide on Titan. Science Advances, 2017, v. 3(7), p. e1700022/6. DOI: https://doi.org/10.1126/ sciadv. 1700022

\section{Georgy S.Bordonsky, e-mail: lgc255@mail.ru}

2. Goesmann F., Rosenbauer H., Bredehöft J.H., Cabane M., Ehrenfreund P., Gautier T., Giri C., Kröger H., Le Roy L., MacDermott A. J., McKenna-Lawlor S., Meierhenrich U. J., Caro G. M. M., Raulin F., Roll R., Steele A., Steininger H., Sternberg R., Szopa C., Thiemann W., Ulamec S. Organic compounds on comet 67P/Churyumov-Gerasimenko revealed by COSAC mass spectrometry. Science, 2015, v. 349(6247), p. aab0689-1/3. DOI: https://doi.org/10.1126/science. aab0689

3. Mumma M. J., Villanueva G. L., Novak R. E., Hewagama T., Bonev B. P., DiSanti M. A., Mandell A. M., Smith M. D. Strong release of methane on Mars in northern summer 2003. Science, 2009, v. 323(5917), pp. 1041-1045. DOI: https://doi.org/10.1126/science. 1165243

4. Korablev O.I. Study of the atmospheres of the terrestrial planets. Physics-Uspekhi, v. 48(6), p. 626- 
635. DOI: https://doi.org/10.1070/PU2005v048n06 ABEH002443

5. Sergeev G. B., Batyuk V.A. Kriokhimiya [Cryochemistry]. Moscow, Khimiya Publ., 1978, 296 p. (in Russ.)

6. Shavlov A. V., Pisarev A. D., Ryabtseva A. A. Dinamika elektroprovodnosti plenok metallov vo l'du pri ego strukturnom prevrashchenii. Rekombinatsionno-fononnyi mekhanizm uskoreniya korrozii [Electroconductivity dynamics of metal films in ice under its structural transformation. The mechanism of corrosion acceleration]. Kriosfera Zemli, 2006, v. 10(3), p. 42-48. (in Russ.)

7. Shavlov A. V., Pisarev A. D., Ryabtseva A. A. Corrosion of metal films in ice: The dynamics of the conductivity of films. Russian Journal of Physical Chemistry A, 2007, v. 81(7), p. 1030-1034. DOI: https://doi. org/10.1134/S0036024407070047

8. Velikotsky M. A. Korrozionnaya aktivnost' gruntov $\mathrm{v}$ razlichnykh prirodnykh zonakh [Corrosive activity of grounds in different natural zones]. Vestnik Moskovskogo Universiteta, Seriya 5: Geografiya, 2010(1), pp. 21-26. (in Russ.)

9. Lotnik S. V., Kazakov V. P. Nizkotemperaturnaya khemilyuminestsentsiya [Low temperature chemiluminescence]. Moscow, Nauka Publ., 1987, 176 p. (in Russ.).

10. Shabatina T. I., Sergeev G. B. Reaktsii pri nizkikh temperaturakh $v$ khimii nanosistem [Low-temperature reactions in the chemistry of nanosystems]. Uspekhi Khimii, 2003, v. 72(7), pp. 643-664. (in Russ.)

11. Chaplin M. Water Structure and Science. Available at: http://www.lsbu.ac.uk/water/chaplin.html (accessed 23.09.2019)

12. Rosenfeld D., Woodley W.L. Deep convective clouds with sustained supercooled liquid water down to $-37.5^{\circ} \mathrm{C}$. Nature, 2000 , v. $405(6785)$, pp. $440-442$. DOI: https://doi.org/10.1038/35013030

13. Bordonskiy G. S., Orlov A. O. Signatures of the appearance of ice 0 in wetted nanoporous media at electromagnetic measurements. JETP Letters, 2017, v. 105(8), pp. 492-496. DOI: https://doi.org/10.1134/ S0021364017080021

14. Limmer D. T., Chandler D. The putative liquidliquid transition is a liquid-solid transition in atomistic models of water. Journal of Chemical Physics, 2011, v. 135 , pp. 134503/10. DOI: https://doi.org/10.1063/ 1.3643333

15. Mishima O.Volume of supercooled water under pressure and the liquid-liquid critical point. Journal of Chemical Physics, 2010, v. 133(14), p. 144503/6. DOI: https://doi.org/10.1063/1.3487999

16. Handle P. H., Loerting T., Sciortino F. Supercooled and glassywater: Metastable liquid(s), amorphous solid(s), and a no-man's land. Proceedings of the National Academy of Sciences USA, 2017, v. 114(51), pp. 13336-13344. DOI: https://doi.org/10.1073/ pnas. 1700103114
17. Speedy R. J., Angell C. A. Isothermal compressibility of supercooled water and evidence for a thermodynamic singularity at $-45^{\circ} \mathrm{C}$. The Journal of Chemical Physics, 1976, v. 65(3), pp. 851-858. DOI: https:// doi.org/10.1073/pnas.170010311410.1063/1.433153

18. Anisimov M. A. Cold and supercooled water: A novel supercritical-fluid solvent. Russian Journal of Physical Chemistry B, 2012, v. 6(8), p. 861-867. DOI: https://doi.org/10.1073/pnas.170010311410. 1134/S199079311208009X

19. Bordonskiy G. S., Gurulev A. A. Experimental proof of the existence of a Widom line based on peculiarities of the behavior of hydrogen in nanoporous silicate at $-45^{\circ} \mathrm{C}$ and atmospheric pressure. Technical Physics Letters, 2017, v. 43(4), pp. 380-382. DOI: https://doi.org/10.1073/pnas.170010311410.1134/ S1063785017040174

20. Bordonskiy G. S., Gurulev A. A., Krylov S. D., Tsyrenzhapov S. V. Using microwave spectroscopy to study the state of supercooled water. Kondensirovannye sredy i mezhfaznye granitsy [Condensed Matter and Interphases], 2019, v. 21(1), pp. 16-23. DOI: https:// doi.org/10.17308/kcmf.2019.21/712 (in Russ.)

21. Castrillyn R. V. S., Giovambattista N., Aksay I. A., Debenedetti P. G. Structure and energetics of thin film water. Journal of Physical Chemistry C, 2011, v.115(11),pp.4624-4635.DOI: https://doi.org/10.1021/ jp1083967

22. Menshikov L. I., Menshikov P. L., Fedichev P. O. Phenomenological Model of Hydrophobic and Hydrophilic Interactions. Journal of Experimental and Theoretical Physics, 2017, v. 125(6), pp. 1173-1188. DOI: https://doi.org/10.1134/S1063776117120056

23. Cerveny S., Mallamace F., Swenson J., Vogel M., $\mathrm{Xu} \mathrm{L}$. Confined water as model of supercooled water. Chemical Reviews, 2016, v. 116(13), pp. 7608-7625. DOI: https://doi.org/10.1021/acs.chemrev.5b00609

24. Gorbatyi Yu. E., Bondarenko G. V. Sverkhkriticheskoe sostoyanie vody [Supercritical state of water]. Sverkhkriticheskie flyuidy: Teoriya i praktika, 2007, v. 2(2), pp. 5-19. (in Russ.)

25. Patashinskii A. Z., Pokrovskii V. L. Fluktuatsionnaya teoriya fazovykh perekhodov [Fluctuation theory of phase transitions]. Moscow, Nauka Publ., 1982, 381 p. (in Russ.)

26. Prigozhin I., Kondepudi D. Sovremennaya termodinamika. Ot teplovykh dvigatelei do dissipativnykh struktur [Modern thermodynamics. From heat engines to dissipative structures.]. Moscow, Mir Publ., 2002, 461 p. (in Russ.)

27. Landau L. D., Lifshits E. M. Teoreticheskaya fizika. Tom. 5. Statisticheskaya fizika. Chast' 1 [Theoretical physics. Tom. 5. Statistical physics. Part 1]. Moscow, Fizmatlit Publ., 2002, 616 p. (in Russ.).

28. Sellberg J. A., Huang C., McQueen T. A., Loh N. D., Laksmono H., Schlesinger D., Sierra R. G., Nordlund D., Hampton C. Y., Starodub D., Depon- 
te D. P., Beye M., Chen C., Martin A. V., Barty A., Wikfeldt K. T., Weiss T. M., Caronna C., Feldkamp J., Skinner L. B., Seibert M. M., Messerschmidt M., Williams G. J., Boutet S., Pettersson L. G. M., Bogan M. J., Nilsson A. Ultrafast X-ray probing of water structure below the homogeneous ice nucleation temperature. Nature, 2014, v. 510(7505), pp. 381-384. DOI: https:// doi.org/10.1038/nature13266.

29. Goy C., Potenza M. A. C., Dedera S., Tomut M., Guillerm E., Kalinin A., Voss K.-O., Schottelius A.,
Petridis N., Prosvetov A., Tejeda G., Fernández J. M., Trautmann C., Caupin F., Glasmacher U., Grisenti R. E. Shrinking of Rapidly Evaporating Water Microdroplets Reveals their Extreme Supercooling // Physical Review Letters, 2018, v. 120(1), p. 015501/6. DOI: https://doi. org/10.1103/PhysRevLett.120.015501

30. Sergeev G. B. Nanokhimiya [Nanochemistry]. Moscow, Knizhnyi dom “Universitet” Publ., 2015, 384 p. (in Russ.)
Бордонский Георгий Степанович - д. ф.-м. н., профессор, зав. лабораторией геофизики криогенеза, Институт природных ресурсов, экологии и криологии Сибирского отделения Российской академии наук, Чита, Российская Федерация; e-mail: lgc255@mail.ru. ORCID iD: https://orcid. org/0000-0002-0009-0822.

Гурулев Александр Александрович - к. ф.-м. н., с. н. с., доцент, Институт природных ресурсов, экологии и криологии Сибирского отделения Российской академии наук, Чита, Российская Федерация; e-mail: sansang@mail.ru. ORCID iD: https://orcid.org/0000-0003-2232-3583.
Bordonsky Georgy S. - Dr. Sci. (Phys.-Math.), Professor, Head Laboratory of Geophysics of Cryogenesis, Institute of Natural Resources, Ecology and Cryology, Siberian Branch of the Russian Academy of Sciences, Chita, Russian Federation; e-mail: lgc255@mail.ru. ORCID iD: https://orcid.org/00000002-0009-0822.

Gurulev Alexander A. - Cand. Sci. (Phys.-Math.), Senior Researcher, Associate Professor, Institute of Natural Resources, Ecology and Cryology, Siberian Branch of the Russian Academy of Sciences, Chita, Russian Federation; e-mail: sansang@ mail.ru. ORCID iD: https://orcid.org/0000-00032232-3583. 